\title{
Introduction to the Mini-track Cross-Organizational and Cross-Border IS/IT Collaboration
}

\author{
Ilsang Ko \\ Chonnam National University \\ isko@chonnam.ac.kr
}

\author{
Daniel Beimborn \\ University of Bamberg \\ daniel.beimborn@uni-bamberg.de
}

Global virtual teams are sharing the benefits of smart collaboration technologies beyond time and space, and even beyond country borders. Smart collaboration, smart services, smart communication, Industry 4.0, IoT applications, and Blockchain use cases were the leading research issues on the 9 submitted papers in our COCB IS/IT collaboration session, this year. Among them, based on the evaluations with the comments and suggestions from 41 reviewers, the 4 papers have been accepted for presentations at HICSS 53.

In our session, smart collaboration in global virtual teams, effectiveness of global help desk team's collaboration, attitude toward security and its relation to compliance intention, communication and conflict in global virtual teams will be the main discussion areas.

The first paper entitled "Smart Collaboration in Global Virtual Teams: The Influence of Culture on Technology Acceptance and Communication Effectiveness" by Carolin Fleischmann, Peter Cardon, and Jolanta Aritz investigates cultural values and practices and their influence on the use of smart communication technology. Team members in individualistic, future oriented culture have shown to have more positive expectations and enjoyment of using the technology.

In the second paper, "Analyzing Performance of a Global Help Desk Team Operation -
Country Handoffs, Efficiencies and Costs" by Akhil Kumar and Siyuan Liu, the authors try to understand the nature of international traffic flows during the resolution of incidents in Volvo IT support help desk. They found that the increasing number of participating countries in help desk services negatively affects IT service efficiency.

The third paper entitled "Cultural Influence and the Effective Use of Security Awareness in Congolese Organizations" by Arnold Nzailu and Insu Park, found that attitudes toward security better explains the effective use of security awareness programs than intention to comply. Culture has also influenced the use of security awareness programs.

The final paper, "Communication and Conflict in Sino-German Global Virtual Teams", by Alexander Jurack, found that video calls bring more amount of task conflicts and cross-cultural conflicts. Social media were proved to mitigate those conflicts.

We would like to appreciate the authors who had submitted their precious papers for review to our COCB IS/IT mini-track on HICSS 53. We would also like to express special thanks to our reviewers for giving valuable comments and suggestions on all the papers that had been submitted to our mini-track. 